\title{
Estructuras Organizacionales y Nueva Ruralidad. El rol de las Cooperativas en el desarrollo territorial rural
}

\author{
Álvarez Montalvo, Amparo* \\ Universidad de las Fuerzas Armadas ESPE \\ E-mail: adalvarez2@espe.edu.ec \\ https://orcid.org/0000-0002-2736-4993
}

Recibido: 17 de diciembre de 2020

Aprobado: 24 de diciembre de 2020

\begin{abstract}
Resumen
Las organizaciones dinámicas se caracterizan por su complejidad, incertidumbre y capacidad para evolucionar y adaptarse a nuevos entornos. En los territorios rurales los cambios gestados en las sociedades modernas han propiciado la aparición de la denominada nueva ruralidad, que articula la tradicional dimensión productiva de agricultura con las restantes (social, institucional, ambiental, educativa y cultural), resultando en un ambiente más dinámico, competido y exigente para las organizaciones participantes. En este escenario, modelos horizontales de organización como las cooperativas retoman importancia para materializar la acción colectiva. Para abordar el tema se diseñó una investigación cualitativa, cuyo objetivo fue contrastar los fundamentos teóricos de las estructuras de organización vertical y horizontal para el desarrollo territorial rural. Los principales aportes revelan la idoneidad de las cooperativas como modelos de organizaciones horizontales más flexibles para la gestión del negocio rural. Estas permiten además alcanzar exitosamente una transición desde la teoría (ideal de la acción colectiva) a la práctica (organizaciones sociales hacia fines comunes específicos), basada en los recursos y especificidades del territorio, así como en la sustentabilidad de las funciones por ellas ejecutadas en el tiempo y en la cohesión comunitaria.
\end{abstract}

Palabras clave: Cooperativas, Desarrollo organizacional, Horizontalidad, Nueva ruralidad, Verticalidad. 


\title{
Organizational Structures and New Rurality. The role of Cooperatives in achieving rural territorial development
}

\begin{abstract}
Dynamic organizations are characterized by their complexity, uncertainty and ability to evolve and adapt to new environments. In rural territories, changes in modern societies have led to the emergence of the so-called New Rurality, which articulates the traditional productive dimension of agriculture with the rest of them (social, institutional, environmental, educational and cultural). As a result, participating organizations must face a more competitive and demanding environment. In this scenario, horizontal models of organization such as cooperatives regain importance to materialize collective action. In order to approach to this subject, a qualitative research was designed, whose objective was to contrast the theoretical foundations of vertical and horizontal organization structures for achieving the rural territorial development. Main contributions reveal the suitability of the cooperatives, as models of horizontal organizations, more flexible for rural business management. This kind of entities also allows to successfully reach a transition from the theory (the ideal of collective action) to practice (social organizations towards specific common purposes), based on the resources and specificities of each territory, the sustainability of the functions thus executed over time and the Community cohesion.
\end{abstract}

Keywords: Cooperatives, Horizontal Organizations, Organizational Development, New Rurality, Vertical organizations.

\section{Introducción}

La sociedad actual es una sociedad de organizaciones, cuya dinámica se caracteriza en líneas generales por la complejidad, la incertidumbre y la capacidad de transformarse y evolucionar para adaptarse a las transformaciones del entorno. En tal sentido, las organizaciones se han abordado como unidades o "extensiones del hombre para alcanzar objetivos difíciles" (Marín, 2013, p. 7).

Las organizaciones también son definidas como agrupaciones humanas o unidades sociales que son construidas (o reconstruidas) de forma deliberada para alcanzar fines específicos (Melinkoff, 1986).

Considerado de forma individual, el término organización ser relaciona en una empresa con la estructura actividad-autoridad. En otros términos, la organización es la agrupación de las actividades necesarias para alcanzar los objetivos de dicha empresa para alcanzar sus objetivos, proceso en el cual cada grupo se ellas es asignado a un ejecutivo que tiene la autoridad requerida para administrarla. Por lo tanto, la organización significa también establecer relaciones de autoridad en la estructura de la empresa, indicando la coordinación tanto vertical como horizontal entre ellas. Por otro lado, la estructura de la organización "es un armazón, un esqueleto, una integración particular, especial y armónica de unidades estructurales de la organización, las cuales mantienen una disposición de interdependencia" (Melinkoff, 1986, p. 48). Se crea para realizar las distintas funciones, actividades y cumplir con los deberes y responsabilidades de sus componentes sociales. A través de ella de delega la autoridad y se establecen las responsabilidades.

Aunque las organizaciones siempre han existido con la humanidad, fue a partir de las sociedades modernas - y concretamente, tras la revolución industrial - cuando las organizaciones comenzaron a tomar verdadera importancia dentro de los procesos de desarrollo económico y social. De otro lado, con frecuencia los estudios sobre la organización en la literatura especializada se basan en teorías provenientes de otras disciplinas de las ciencias sociales, entre otras razones, debido a que los investigadores en este campo justamente van perdiendo de vista a la propia organización. Paradójicamente son pocos los investigadores de las disciplinas afines que son realmente conscientes 
sobre los hallazgos de estos estudios sobre la organización y sus implicaciones (Ahrne, Brunsso y Seidl, 2017). En este contexto, los autores proponen (desde los clásicos de los estudios de organización) conceptualizar a la organización como un orden social decidido, extendiendo dicho concepto -pero ahora trascendiendo a los clásicos- más allá de las organizaciones formales, por cuanto en realidad existen órdenes sociales definidos que se hallan fuera de la definición de organizaciones formales.

Para Marín (2013) las organizaciones son "un tipo de agrupación de los individuos muy diferente de las familias y grupos en general" (p. vii). Por lo tanto, son unidades conformadas por grupos de individuos que cumplen con un conjunto de funciones sociales vinculadas con la comunicación, la producción y comercialización de bienes y servicios, entre otras. Una forma de organización "típica es la empresa en la que los objetivos planteados por las personas integradas en ella son fundamentalmente económicos" (Marín, 2013, p. 15).

En tal contexto destaca la existencia de diversas teorías para el abordaje de algunos aspectos clave de la organización, tales como los modelos de gestión, la cultura, el desarrollo organizacional y la estructura. Esta última es precisamente el elemento de interés que orienta este artículo, en tanto permite abordar el desarrollo de las organizaciones a partir de los cambios introducidos en su estructura. Como ha sido reseñado ampliamente en la literatura, inicialmente -a partir de los postulados de la teoría clásica- se extendió el enfoque burocrático (como modelo generalizado para el análisis organizacional, tanto en la teoría como en la práctica), cuya estructura mecanicista, vertical y centralizada resultaba como la más adecuada para el alcance de los objetivos organizaciones en los términos de eficiencia de sus miembros para el desarrollo de las actividades y funciones.

No obstante, a partir de la evolución de las sociedades y la transformaciones ocurridas (acentuadas de manera particular a partir de la globalización), la teoría sobre las organizaciones ha ido evolucionado para adoptar más recientemente "planteamientos más enfocados al papel insustituible de las personas individuales en la buena marcha de las empresas" (Marín, 2013, p. 348). Han surgido así nuevos enfoques, como el de las estructuras horizontales, cuyos postulados promueven la conformación de estructuras organizacionales más planas y más flexibles que resultan más adecuadas para promover el desarrollo organizacional en todas sus dimensiones.

En el ámbito del desarrollo rural, a partir de la revisión de literatura especializada se encuentra que a principios de la década de 2000 y en cuanto a los progresos sobre una teoría para el desarrollo rural, el énfasis estuvo en el debate desde un enfoque territorial de este último. Hubo entonces algunas iniciativas para abordarlo desde una perspectiva integral, interdisciplinaria y sistematizada. Destacan aquí las contribuciones de Schejtman y Berdegué (2004), para quienes el desarrollo rural con visión territorial es concebido como un proceso de transformación tanto de la dinámica productiva como de lo institucional, que tiene lugar en un ámbito rural determinado con el fin último de reducir los índices de pobreza rural.

Desde esta visión de la ruralidad el cooperativismo aparece como una forma de organización colectiva que permite gestionar con criterios de eficiencia y sustentabilidad los recursos territoriales, para alcanzar objetivos comunes vinculados con la reducción de la pobreza rural y el mejoramiento de la calidad de vida de la población. De hecho, en las últimas décadas las cooperativas agrícolas han cambiado considerablemente, rediseñando su estructura tradicional y adoptando modelos no tradicionales como respuesta a los cambios en sus entornos competitivos e institucionales (Chaddad e Iliopoulos, 2012). Uno de estos, dentro de las cadenas de valor globales, es el cambio desde una orientación a la producción hacia una estrategia orientada al mercado, impulsada por una demanda creciente del consumidor por una mayor calidad y una mayor variedad de productos (Beber, Theuvsen y Otter, 2018).

Sobre la base de los argumentos antes descritos, este artículo tiene como objetivo contrastar los fundamentos teóricos de las estructuras verticales de 
organización vs. las horizontales, con énfasis dentro de estas últimas del cooperativismo en el contexto del desarrollo territorial rural. Metodológicamente se adoptó un diseño bibliográfico, sustentado en la revisión de literatura especializada en las líneas de investigación vinculadas con investigación sobre organizaciones, sociología de las organizaciones, estructuras burocráticas y horizontales, multifuncionalidad de la agricultura, desarrollo territorial rural y cooperativismo.

El artículo se estructuró en cinco secciones que incluyen la presente introducción. La segunda analiza el tema de las organizaciones burocráticas (verticales) yeldesarrollo organizacional, destacando sus contribuciones y limitaciones para promover las transformaciones organizacionales requeridas para que estas puedan insertarse competitivamente en mercados globalizados. La tercera enfatiza en la importancia de las organizaciones horizontales como estructura alternativa que, por sus características y flexibilidad, favorecen el desarrollo de las organizaciones en entornos complejos, dinámicos y altamente competitivos. La cuarta analiza las relaciones entre desarrollo territorial rural y las cooperativas en tanto modelo de organización horizontal idóneo para alcanzar en la práctica los objetivos del desarrollo rural. Finalmente se presentan, a manera de conclusión, algunas reflexiones finales sobre estos aspectos y sus interrelaciones como modelo alternativo orientado a alcanzar dicho desarrollo desde el territorio.

\section{Organizaciones burocráticas desarrollo organizacional}

En el campo académico el debate sobre las organizaciones se ha centrado en los mecanismos de control adoptados como un instrumento para promover el desempeño de las actividades con criterios de eficiencia. El control se ha analizado desde diversas posturas destacando las propuestas de la teoría burocrática, la teoría de la agencia, la teoría de los sistemas sociales, entre otras. En esta sección se abordan los postulados básicos que sustentan la comprensión de las organizaciones desde un enfoque basado en las estructuras de control burocráticas o tradicionales, así como sus principales limitaciones para el mejor desempeño y competitividad de las organizaciones en un ambiente más global, dinámico y complejo.

Con respecto a las organizaciones burocráticas Morgan (1990) afirma que éstas se han concebido como "organizaciones mecanicistas". Para el autor, sus orígenes se remontan a la Revolución Industrial donde se partió de la premisa que para el buen funcionamiento de las máquinas -especialmente en el sector industrial-, se requería que las organizaciones se adaptaran a sus necesidades, lo cual indujo una "rutinización" de las actividades, tareas y funciones organizacionales. Tal situación se evidenció en el mundo de las organizaciones por parte de sus propios dueños e ingenieros, encontrándose que el desempeño eficiente de tales entidades se alcanzaba cuando se adoptaban acciones para mejorar la distribución del trabajo entre sus miembros (Morgan, 1990).

Los postulados de Weber (citado por Hall, 1996) aportaron las bases para definir la burocracia como una estructura que tiene "jerarquía de autoridad, autoridad limitada, división de labores, participantes técnicamente competentes, procedimientos para el trabajo, reglas para los ocupantes de puestos y compensaciones diferenciadas" (p. 54). Parte de la idea de que cuando las características previamente mencionadas se manifestaban en la organización, ésta se encontraba en capacidad de alcanzar el modelo "ideal de burocracia".

A los rasgos antes expuestos Perrow (1991) agrega los siguientes: a) la igualdad de trato para todos los empleados; b) las habilidades, pericias y experiencia como aspectos clave para la asignación de los puestos de trabajo; c) la adopción de criterios de eficiencia en el trabajo y en la productividad como base para el desarrollo de la organización; d) la importancia del control en el desempeño de las actividades; y e) el cumplimiento obligatorio de leyes, regulaciones y normas por parte de directivos y empleados como mecanismo que garantice los intereses de la organización.

En relación con la estructura de las organizaciones, Marín (2013) expone que se trata de un aspecto que

Álvarez Montalvo, Amparo 
va más allá de las relaciones sociales para abarcar "las relaciones entre los roles -posiciones y tareasque desempeñan las personas en el seno de una organización" (p. 272). De esta manera, dentro de las organizaciones burocráticas destaca la adopción de una estructura de jerarquías "vertical", que permite distinguir los niveles de autoridad en la organización. Esto significa que (la más alta jerarquía, los gerentes y supervisores) dispone de algunos derechos tales "como la capacidad de recompensar y castigar por el cumplimiento de las propias tareas" (Marín, 2013, p. 273).

Estas bases de la estructura de organizaciones "verticales" son reafirmadas con la adopción de los principios de la administración científica propuestos por Taylor (citado por Morgan, 1990) y que pueden ser resumidos en: a) los directivos tienen la función de planificar y organizar el trabajo que realizarán los empleados; b) la adopción de métodos científicos para determinar el mecanismo más eficiente para el desarrollo del trabajo; c) la selección de la "mejor" persona para el desempeño del cargo; d) la instrucción del trabajador para el desarrollo de las actividades con criterios de eficiencia; y e) el establecimiento de controles para monitorear el rendimiento del trabajador.

El modelo descrito se ha fundamentado en la definición de rangos al nivel de cada organización, donde los cargos "que ocupa la gente tienen reglas y reglamentos que especifican, en diferentes grados, cómo deben comportarse los que ocupan estas posiciones" (Hall, 1996, p. 53). Esta estructura organizacional contribuye con el cumplimiento de tres funciones básicas, a saber: a) alcanzar los objetivos de la organización; b) reducir la influencia de las conductas individuales sobre el desempeño de la organización; y c) promover el ejercicio del poder.

Los fundamentos del modelo burocrático han contribuido con el posicionamiento de algunas organizaciones en los mercados internacionales. Tal es el caso de las cadenas de comida rápida que han adoptado los principios del taylorismo para la organización de la producción y del trabajo. Al respecto, Morgan (1990) y Ritzer (1996) analizaron el desempeño de la corporación McDonalds, cuyo modelo de gestión se ha fundamentado en los postulados propuestos por Taylor, que le han permitido la mecanización de sus procesos y la estandarización de los productos comercializados en los establecimientos expandidos a nivel mundial (bajo un modelo de franquicias).

La empresa se ha caracterizado por el empleo de los "procedimientos de producción en cadena para cocinar y servir comida" (Ritzer, 1996, p. 48), prestando un servicio regular y constante de un conjunto de productos "con toda la exactitud que puede dar lugar a la ciencia de la hamburguesa" (Morgan, 1990, p. 24). Esto significa que cualquier consumidor en cualquier parte del mundo puede entrar a un establecimiento de la Corporación, con la certeza de que el producto que adquiera es estandarizado (tendrá casi las mismas características organolépticas y calidad, bajo los mismos procedimientos -e incluso vendido al mismo precio, dando lugar al mundialmente conocido "Índice Big Mac" o McDólar-, sin importar dónde se hace). Además, la innovación que pueda tener lugar en alguno de los países o franquicias en los que está presente, si es exitosa será replicada en el resto de ellas. Desde el punto de vista laboral, se reclutan jóvenes estudiantes y universitarios que rápidamente se identifican con la cultura organizacional de la Corporación. Tales aspectos son claves para que McDonalds se desempeñe como una organización mecanicista donde "la máquina de trabajo funciona perfectamente la mayoría de las veces" (Morgan, 1990, p. 24).

La base del éxito alcanzado por McDonalds pudiera encontrarse en el planteamiento de Hall (1969), al afirmar que las organizaciones burocráticas en el mundo real se han diseñado para ser eficientes y confiables, presentándose variaciones en sus modelos de gestión y concretamente en el modelo burocrático "ideal", en función de la naturaleza, rasgos y especificidades de cada organización en particular. En contraste a esta postura, Perrow (1998) ha señalado que el modelo burocrático no se presenta en la práctica de "manera ideal", pues sus bases: a) intentan eliminar las influencias de los factores externos a la organización sobre el desempeño de sus miembros, en la medida que sus 
acciones se limitan a garantizar los intereses de la misma; b) las burocracias se crean para administrar y controlar el desarrollo de tareas rutinarias y estables que contribuyen con la materialización de algunos objetivos predefinidos; $y \mathrm{c}$ ) las burocracias crean unas expectativas irrealizables, que no se materializan en la práctica, pues las organizaciones se conforman para personas "medianamente inteligentes" que desarrollan todas sus actividades en los puestos de trabajo. En otras palabras, los individuos dentro de este tipo de organizaciones no son más que medios que solo cumplen funciones rutinarias para alcanzar los objetivos de aquélla, quedando por lo tanto al margen otros aspectos sociales como sus aspiraciones, crecimiento personal, motivaciones, creatividad y capacidad de innovación, entre otras.

Tal posición es complementada por Etkin (2005) al plantear que en el mundo real las organizaciones no se conciben solamente como construcciones orientadas hacia el alcance de objetivos predefinidos, donde sus miembros desempeñan sus actividades como una especie de engranajes "de una máquina o partes pasivas de un sistema ('componentes')" (p. 8). No obstante, la realidad de la práctica organizacional demuestra que las organizaciones están conformadas por individuos que tienen sus propias necesidades y cuya racionalidad no se limita al cumplimento de programas, normas y regulaciones, pues sus acciones resultan fundamentales para promover el desarrollo organizacional.

Entre las desventajas del modelo burocrático Perrow (1998) enfatiza que la estructura organizacional basada en jerarquías verticales -debido a su carácter rígido- limita la toma de decisiones, pues los empleados de menor jerarquía -o sea, los subordinados- no se encuentran en la capacidad ni tienen la motivación para sugerir los cambios que pudieran inducir al mejoramiento en el desempeño de la organización. Para el autor, la jerarquía genera retrasos e inercia en la organización, en la medida que las decisiones se toman al nivel superior (directivos, gerentes y supervisores), mientras los empleados no tienen la autorización para tomar decisiones, aun cuando éstas puedan contribuir con el cumplimiento de los objetivos de la organización de manera más eficiente.
La situación descrita ha limitado el desarrollo de las capacidades y competencias de los miembros de la organización para promover el cambio, pues el modelo burocrático -cuyo énfasis está en las estructuras jerárquicas-, el poder y el control sobre las actividades y las personas resultan insuficientes para la toma de decisiones. Esto precisamente revela la necesidad de adoptar otros tipos de estructuras, caracterizadas por su capacidad de promover las transformaciones que la organización requiere para adaptarse a sociedades más dinámicas y complejas. En esta dinámica han emergido las estructuras horizontales como vertiente alternativa, que por ser más planas y más flexibles favorecen el desarrollo de la organización en sus distintas dimensiones, aspecto que será discutido en la siguiente sección.

\section{Organizaciones horizontales como modelo alternativo para el desarrollo organizacional}

En el ámbito de la economía real (i.e., los negocios y las organizaciones en la práctica), desde hace décadas han aparecido y se han consolidado nuevas formas organizativas, basadas tanto en estructuras alternativas al modelo burocrático como en prácticas de dirección que en cierta medida intentaron paliar o corregir las limitaciones derivadas de su adopción. Ejemplos de ello han sido iniciativas como la gerencia con puertas abiertas, el enfoque de la calidad total (Ishikawa, 1986), el justo a tiempo (Ohno, 1988), las iniciativas jointventure (Pearce, 1997), las redes de empresa, el comportamiento complejo (Navarro, Roe y Artiles, 2015), la teoría de la agencia (Ganga, Quiroz y Maluk, 2015), el enfoque de capacidad absorbente (Mariano y Al-Arrayed, 2018), entre otras.

También han cobrado mayor importancia los modelos de conglomerados, basados por ejemplo en las fusiones, absorciones y adquisiciones -en particular,comopartedelosefectosdelaglobalización sobre el desempeño de la organización-, en última instancia orientados a mejorar la competitividad de tales organizaciones, al tiempo que se encontraban mecanismos y fuentes alternativas para generar e incrementar el valor creado a través de los productos y servicios ofrecidos; todo ello en un mercado no 
solo más competido, sino con consumidores más informados y comprometidos con la sociedad y el planeta y marcos institucionales -locales, regionales y globales- cada vez más exigentes en aspectos como la sustentabilidad ambiental, la equidad de género, la responsabilidad social corporativa, entre otros. Como resultado, las estructuras tradicionales de gestión de los negocios y de las organizaciones en cualquier sector económico terminaron siendo insuficientes e incapaces de dar respuesta a estos nuevos desafíos.

Ejemplo exitoso de este tipo de organizaciones son las cooperativas, que no obstante su antigüedad, están actualmente presentes en casi todos los países del planeta y en todos los sectores económicos. Se trata de entidades cuya estructura funcional de tipo horizontal les ha convertido en alternativas viables en el nuevo escenario global.

En líneas generales, las cooperativas -entendidas como asociaciones autónomas y voluntarias de personas en una empresa o iniciativa de propiedad conjunta y de gestión democrática-, a nivel internacional se rigen por los llamados "principios cooperativistas". Los mismos fueron establecidos en "La declaración sobre la identidad cooperativa" (Alianza Cooperativa Internacional, 2013) y pueden resumirse en los siguientes términos: 1) adhesión voluntaria y abierta: apertura para todos aquellos quienes así lo deseen y que además tengan la capacidad y disposición para utilizar sus servicios y aceptar las responsabilidades derivadas de convertirse en asociado, sin ningún tipo de discriminación; 2) control democrático de los miembros: si bien depende del grado de la cooperativa, la fijación de sus políticas y la toma de decisiones se realiza con la participación de todos los asociados; algunos son elegidos para representar y gestionar los intereses del colectivo ("agentes") y por lo tanto son responsables ante ellos de su gestión; 3) participación económica de los miembros: en general, todos los asociados contribuyen con el capital de su cooperativa, que -al menos en parte- es propiedad común o colectiva de la entidad.

Por lo tanto, los asociados son compensados -en caso de excedentes económico-financieros positivos de la gestión-, en proporción al capital entregado; 4) autonomía e independencia: al ser gestionadas por sus asociados $-\mathrm{o}$ los agentes nombrados por estos- y ser organizaciones de autoayuda, aunque establezcan acuerdos con otras organizaciones públicas o privadas -incluidos gobiernos y entidades comunitarias-, el control democrático se mantiene en manos de los asociados y deciden sobre la base de la autodeterminación; 5) educación, formación e información: con el fin de alcanzar de manera eficaz y eficiente sus objetivos, estas entidades proporcionan tanto a sus asociados como a sus representantes (agentes), directivos y empleados, capacitación y educación; 6) cooperación entre cooperativas: para alcanzar tales objetivos también se integran con otras estructuras ya sean locales, nacionales, regionales e internacionales, así como para fortalecer el movimiento cooperativo; y, 7) interés por la comunidad: mediante las acciones de sus asociados se preocupan y actúan para alcanzar el desarrollo sostenible de las comunidades de las cuales forman parte indisoluble.

De todos ellos, son fundamentalmente tres los que aportan las bases para poder hablar de una gestión horizontal: el de adhesión voluntaria y abierta; el control democrático de los miembros y el de autonomía e independencia. En relación con el primero y aunque también en las organizaciones tradicionales (entre ellas, las empresas con fines de lucro) puede haber adhesión voluntaria -un individuo puede decidir si trabaja o no para una entidad, o si compra o no acciones de la misma-, la Gerencia, o la Directiva -en general, cualquiera de los órganos de dirección- puede decidir iniciar o cesar la relación de trabajo; e incluso, adelantar acciones -amistosas u hostiles- para propiciar la salida de determinados accionistas o grupos de ellos, de acuerdo con determinados intereses de un subgrupo. En contraste, generalmente en las cooperativas -donde lo que une a los accionistas es justamente es la existencia de un fin o interés colectivo-, este tipo de decisiones se toman en las asambleas de socios y por lo tanto son decisiones del colectivo.

Con respecto al segundo, en las organizaciones verticales el control de la entidad -y por lo tanto, de 
las decisiones inherentes a su funcionamiento- está en manos de un grupo reducido, que generalmente se corresponde con los accionistas mayoritarios. $\mathrm{Si}$ bien excepcionalmente esto podría ocurrir en algunas cooperativas, en la mayoría de ellas priva el control del colectivo sobre todas las decisiones relevantes para su desempeño, en la que cualquiera de los asociados tiene la posibilidad real de participar en la toma de tales decisiones. Así, la horizontalidad permite la participación activa en los cursos de acción de la cooperativa, que en el caso de las cooperativas de Primer Grado ("un socio, un voto"), ocurre de forma igualitaria. En contraste, la estructura vertical tradicional prácticamente puede llegar a ignorar las opiniones o propuestas de acción de los grupos minoritarios, carentes de poder.

Finalmente, con respecto al tercero (autonomía e independencia) es necesario tener en cuenta que los modelos de gestión de negocios en la actualidad -incluso en las cooperativas- tienden a emular los modelos exitosos de gerencia adoptados por las organizaciones tradicionales. Así, cooperativas muy pequeñas o gigantes trasnacionales cada vez más recurren a la contratación de servicios gerenciales, de mercadeo y otros, gestionados por profesionales en dichas áreas y con conocimientos profundos sobre el mercado, competidores y cualesquiera de las variables relevantes para garantizar el éxito de la actividad y el cumplimiento de sus objetivos.

No obstante, a diferencia de la estructura vertical -organizaciones en las que las decisiones de estos profesionales están sujetas a los intereses y directivas de apenas una parte de los accionistas, propietarios de la mayoría del capital, pero que no representan a todos los grupos de interés-, las decisiones adoptadas en las cooperativas -por su naturaleza, arriba explicada- representan y protegen el interés del colectivo. Esto último representa una ventaja frente a las organizaciones tradicionales, pues al privar el interés social y de todo el colectivo, se reducen los riesgos e incentivos para la ocurrencia de fraudes, manejos dolosos y situaciones que se traduzcan en gestiones financiero-económicas deficitarias (riesgo moral). Así, si bien no son los únicos, estos principios confieren a la cooperativa lo que en la literatura se denomina "flexibilidad" organizacional, es decir, mayor capacidad de adaptación y respuesta a las demandas del entorno dinámico y complejo en el que se desenvuelven.

De manera que, tal y como apunta la teoría de la organización, el cambio efectivo en este (y en cualquier otro) tipo de organización se puede lograr si se modifica la mentalidad de sus miembros para que sus comportamientos se alineen con los objetivos y rutinas de ella (Tucker, Hendy y Barlow, 2016). Por lo tanto, el desafío está en promover, desde las propias entidades cooperativas o desde fuera (e.g., organismos de la Administración Pública/Estado, ONG, cooperación internacional, entre otros), un cambio en la percepción de los asociados sobre cómo actúan y cómo pueden mejorar el desempeño sus cooperativas. Para ello fomentar la innovación y garantizar la eficiencia son claves esenciales para lograr un desempeño organizativo sostenido (Lukoschek et al., 2018).

\section{Desarrollo territorial rural y cooperativismo como modelo de organizaciones horizontales exitosas}

\subsection{Lo rural, la ruralidad y el desarrollo los territorios rurales}

Cuando se hace referencia a "lo rural" $-\mathrm{y}$ específicamente en el caso del desarrollo rural o desarrollo territorial rural-, en necesario tener en cuenta que lo rural trasciende lo estrictamente agropecuario, estableciendo al mismo tiempo fuertes vínculos de intercambio con lo urbano. De manera que la función de un espacio rural ya no es solo la de proveer alimentos, sino también satisfacer la demanda creciente de una diversidad de bienes y servicios (tales como recursos naturales, espacios para el esparcimiento, contribución al mantenimiento y desarrollo de la cultura) (Pérez, 2001).

Por su parte, Baudel (2001) expone que lo rural se presenta en un espacio determinado, configurándose -en primer lugar- a partir de un territorio construido socialmente en función de las diversas formas de ocupación del mismo y de dominación social. Tales formas tienen su origen en la estructura de la propiedad; en el uso de la tierra y de los recursos

Álvarez Montalvo, Amparo 
naturales; los paisajes y las relaciones que se establecen entre lo urbano y lo rural. En segundo lugar, lo rural se presenta como un "lugar de vida", para referirse a las diversas formas de vida que coexisten en el medio rural, las cuales contribuyen con la construcción de identidad.

La concepción tradicional de ruralidad consideraba que solo la ciudad podía entregar bienestar a sus habitantes, por lo que alcanzar el bienestar prácticamente es imposible para la mayoría de los habitantes rurales. De esta manera existía un "sector" rural que venía a ser una construcción social residual sobre lo que se define positivamente como lo urbano y lo moderno. Esta concepción de la ruralidad tradicional lleva a una sobrevaloración de lo urbano, pues el concepto mismo de desarrollo -asociado al progreso- llevaba una dirección única: del campo a la ciudad, desde lo rural hacia lo urbano, de la agricultura hacia la industria. Tal premisa fue propuesta y desarrollada ampliamente por Johnston y Mellor (1962), quienes señalaban que el desarrollo económico tenía entre sus bases la migración de la mano de obra de la agricultura a la industria.

Adicionalmente, la concepción tradicional tampoco daba cuenta de ciertos fenómenos contemporáneos, tales como la diversidad de ocupaciones y de situaciones que tienen lugar en el espacio rural, la multiplicidad de actividades productivas y de servicios (estando muy pocos de ellos ligados a la agricultura y cuya importancia es decreciente en el tiempo) o a los efectos de la globalización sobre tales espacios (Gómez, 2001).

Estudios más recientes sobre el desarrollo territorial (como por ejemplo, Gallo, 2009) han evidenciado cómo el uso de este enfoque se ha extendido hasta diversas instituciones u organismos internacionales vinculados por su naturaleza y diseño con los temas rurales; son los que han adoptado las bases del desarrollo territorial rural cuando asumen sus papel para diseñar e implementar políticas y estrategias de desarrollo. Es el caso de la Organización de las Naciones Unidas para la Agricultura y la Alimentación-FAO, el Instituto de Cooperación para la Agricultura-IICA, la Comisión Económica para América Latina y el Caribe-CEPAL, el Banco Mundial-BM, el Banco Interamericano de Desarrollo-BID y el Instituto Latinoamericano y del Caribe de Planificación Económica y SocialILPES, entre otros. Más recientemente aparece en la literatura la denominada "Nueva Ruralidad", corriente bajo la cual el desarrollo es entendido como un proceso orientado al mejoramiento del bienestar tanto de la población rural como urbana y básicamente sustentado en los recursos naturales (Ceña, 1993). Por lo tanto, se ha puesto en relieve la necesidad de revalorizar lo rural, dado que se concibe como una entidad socioeconómica cuyas funciones son trascendentales tanto para la sociedad como para promover y alcanzar el desarrollo económico (Pérez, 2001).

Basados en los argumentos aquí reseñados se puede decir que el desarrollo rural desde el territorio añade a la tradicional dimensión económica (o productiva) de la ruralidad otras de tipo social, institucional, ambiental, educativa y cultural, que también están presentes en el territorio (Tizon, 1996). Como resultado, el territorio se concibe ahora como un espacio abstracto, caracterizado por una dinámica social y en donde se promueve una idea o un proyecto de desarrollo concertado por los diferentes actores sociales ligados a él geográficamente, orientado a la utilización de los recursos territoriales para proponer soluciones en función de sus condiciones y características territoriales (Abramovay, 2006; Schejtman \& Berdegué, 2004).

Adicionalmente es necesario recordar que la pérdida de importancia de la agricultura en aspectos como la cantidad de mano de obra empleada y la creación de riqueza en los llamados países desarrollados no necesariamente se tradujo en una reducción proporcional de la relevancia de las áreas rurales en la vida nacional (Abramovay, 2007). De acuerdo con el autor, la explicación está en el hecho de que en esos países - a diferencia de muchos países en desarrollo, término ahora sustituido por otros como economías emergentes o bloques regionales; en este caso, los Latinoamericanos- la ruralidad es definida sobre la base de un criterio territorial, geográfico y relacionado con la manera cómo viven las personas. De allí que se consideren como áreas o espacios rurales a aquellas que tienen una densidad 
demográfica relativamente baja y que se hallan situadas muy cerca de ecosistemas que han sido alterados relativamente poco por la acción humana.

Desde esta perspectiva se concluye que la agricultura -considerada en sentido amplio, en este caso equivalente a la dimensión económica o productiva- ya no es lo único que define al espacio rural. De hecho, el número personas ocupadas en dicha actividad se ha reducido significativamente, al tiempo que paradójicamente aumenta el número de residentes en el campo o espacio rural. Estos desarrollan cada vez más actividades del tipo no agrícola, al tiempo que aparece un importante número de pequeños agricultores que combinan la agricultura con otras fuentes de ingresos (Graziano da Silva, 1996). Ha sido así como estas áreas pasaron de ser fundamentalmente proveedoras de materias primas, a convertirse en los sitios en donde se instalaron ingentes industrias al finalizar II Guerra Mundial, para más tarde ser también lugares de recreación, que atraen a las poblaciones de jubilados o proveedoras de servicios ambientales (Abromavay, 2007).

El proceso descrito es lo que diversos actores llaman la multifuncionalidad o pluriactividad de la agricultura, o lo que es lo mismo, la multiplicidad de actividades económicas y socioculturales que tienen lugar en los territorios rurales: procesos de producción, como por ejemplo, la generación conjunta de bienes públicos y privados, que son relevantes para cumplir los objetivos que se ha fijado la sociedad (Reig, 2007). No obstante, la pérdida de importancia de la agricultura y el trabajo agrícola en el mundo rural no es exclusiva de países desarrollados, sino más bien un fenómeno generalizado al nivel internacional, aunque obviamente ocurre a un ritmo más o menos acelerado según el caso (Abramovay, 2007).

Es importante advertir que la multifuncionalidad de la agricultura en ocasiones se ha entendido por algunos autores como un sector productivo (o sea, la producción primaria que allí ocurre), mientras que para otros se ha entendido desde una perspectiva basada en el territorio, o sea, la multifuncionalidad de los espacios rurales (Reig, 2007). Por lo tanto, el desarrollo rural no puede ser concebido bajo una óptica sectorial o unidimensional, en los que la expansión de la agricultura sea al mismo tiempo la condición necesaria y suficiente. Así, la ruralidad es una noción de naturaleza territorial que, por definición, abarca diversos sectores económicos (Abramovay, 2007).

De manera que al hablar del desarrollo territorial está implícito que se trata de un enfoque multidimensional, bajo el cual se integran -entre otros- una determinada proporción de actores económicos, productivos y sociales, para elevar el bienestar de los productores. Esto debe ocurrir simultáneamente con el desarrollo rural sustentable, que considera sus diferentes dimensiones: capital humano, físico, social, económico y financiero (Retes et al., 2017).

A pesar de las particularidades y aparentes controversias antes explicadas, es la flexibilidad inherente del concepto de multifuncionalidad la que permite establecer puentes entre los enfoques sectoriales y territoriales de la agricultura, al tiempo que contribuye a superar determinadas imprecisiones y ambigüedades ampliamente debatidas en la literatura sobre el tema (Silva, 2010). Esta autora asoma una variante del concepto a la que denomina desarrollo territorial integrado, que concibe como una oportunidad para materializar "los puentes". En este orden de ideas, el desarrollo debe entenderse en términos de dinámicas que conducen a superar los desequilibrios, respetando las diferencias y particularidades territoriales; esto implica -desde el punto de vista espacialconsiderar simultáneamente al territorio como un todo e involucrarse en las particularidades de cada una de sus partes. Se conforma así una aproximación diferente y complementaria a la planteada en los estudios sobre la multifuncionalidad, que en este caso sitúa al territorio en el eje del análisis.

\subsection{Organizaciones horizontales para el desarrollo territorial rural: el caso de las cooperativas}

A juicio de Carneiro (1998) los trabajos de investigación realizados en los países desarrollados

Álvarez Montalvo, Amparo 
(antes de la llamada "nueva ruralidad") han demostrado algunos hechos que merecen ser destacados. Uno de ellos ha sido la rápida adaptación del agricultor al proceso de modernización (en su caso, en referencia al actor de la agricultura familiar), con técnicas avanzadas y contribuyendo desde su cultura con la formulación de respuestas a la crisis del modelo productivista, como por ejemplo a través de la pluriactividad. Por lo tanto, en lugar de hablar de la cultura campesina como un contraste frente a otra "cultura urbano-industrial" (tal y como ocurre con la llamada "descampesinización" del campo), algunos autores ya entonces señalaban la necesidad de romper con esta referencia para evaluar los verdaderos cambios que el agricultor familiar - una categoría social amplia y genérica- ha experimentado a lo largo del tiempo.

Con respecto a países como la (antigua) Alemania Occidental, destaca la capacidad de la cultura campesina tradicional para salir de las crisis de la economía y del ambiente provocadas por las medidas modernizadoras (Pongratz, citado por la autora). Así, mantener tradiciones culturales "campesinas" no sería incompatible con la modernización de la sociedad, si bien ocurriría en una nueva fase en la que se definan nuevos objetivos, pero incluyendo también los elementos tradicionales.

En el escenario de la nueva ruralidad y la multifuncionalidad de la agricultura algunas formas de organización colectiva cobraron nuevamente importancia. Algunos autores (como Hellin, Lundy y Meijer, 2009) señalan que la organización de agricultores y la acción colectiva son considerados frecuentemente como factores clave para mejorar el acceso de los agricultores a los mercados ${ }^{1}$. Acción colectiva se refiere aquí a la actuación que de forma voluntaria exhibida por un grupo para alcanzar o materializar intereses comunes. No obstante, en el caso de la agricultura, la acción colectiva puede ocurrir aún en ausencia de organizaciones formales por parte de los agricultores. Pero éstas son precisamente la forma cómo se expresa más formalmente dicha acción y pueden tales organizaciones pueden ser las proveedoras de una amplia gama de servicios que son fundamentales para poder materializar el acceso a los mercados.

El problema es que el proceso de establecer organizaciones viables no es tan simple como parece. De hecho, ciertos aspectos organizativos tales como el establecimiento de reglas acordadas colectivamente, el asegurar los compromisos de sus miembros de cumplir con dichas reglas y el hacerlas cumplir y monitorear su cumplimiento pueden representar un verdadero desafío para quienes acometen este tipo de tareas. Además, en algunos casos se incurre en costos de transacción tales que los beneficios de la acción colectiva y la organización de los agricultores para reducir (los costos de transacción) no superan a los beneficios; o que muchas veces los agricultores o pobladores rurales carecen de los recursos necesarios para materializar una cooperación exitosa (como, por ejemplo, de educación básica, de habilidades de gestión y empresariales, de capacidad financiera, entre otras) (Hellin, 2009). Por lo tanto, pasar de la teoría a la práctica, de manera exitosa, no siempre ocurre; o al menos, no ocurre con la frecuencia deseada.

Frente a estas nuevas realidades en el ámbito rural, los modelos de organizaciones horizontales como las cooperativas podrían constituir justamente la forma de transición entre la teoría (el ideal de la acción colectiva) y la práctica (organizaciones orientadas a fines específicos comunes), en la medida que al conformarse como empresas sociales integran las dimensiones de lo rural, de lo político y de lo social (Steiner y Teasdale, 2017). Pero además este tipo de organizaciones reconoce la interconexión entre las diferentes necesidades y demandas en el ámbito rural y sus posibles soluciones. De esta manera, los desafíos allí presentes pueden

1. Con frecuencia suele citarse a la Danesa Arla Foods como ejemplo de cooperativa que ha llegado a insertarse de manera exitosa en los mercados internacionales, tanto como parte de su estrategia de crecimiento (internacionalización, que supone además más mercados para la producción de sus asociados), como en la búsqueda de nuevos socios en tales mercados (Juliá, 2011).

REVISTA DE INVESTIGACIÓN SIGMA / Vol. 07, Nº 2, 2020 (pág. 48-63) 
convertirse en oportunidades para la creación de las cooperativas y su consolidación en los procesos de desarrollo de la comunidad (cohesión comunitaria), así como alternativas que permitan reorientar la oferta de servicios públicos (mejor adaptados a las especificidades de cada contexto rural), como un enfoque integrado dirigido a enfrentar problemas de naturaleza local desde las propias comunidades (descentralización de funciones y empoderamiento de los actores locales).

No obstante, también advierten que activar ese potencial que tiene las cooperativas en el desarrollo rural implica adicionalmente transcender de los enfoques tradicionales (es decir, aquellos que tratan al desarrollo económico como compartimientos estancos o considerando a sus distintos elementos de manera aislada o desconectada, entre ellos cohesión comunitaria y los servicios públicos), que es precisamente una de las debilidades de las políticas públicas diseñadas desde el nivel central (o nacional) y que no siempre se trasladan en la práctica a nivel rural (Steiner y Teasdale, 2017).

Otra ventaja de las cooperativas como modelos de gestión es su flexibilidad: pueden adaptarse tanto para explotaciones a gran escala (también llamadas en la literatura corporaciones agropecuarias capitalistas), así como a las explotaciones independientes (de menor envergadura), al igual que pueden servir como medios para que los agricultores (originalmente independientes) puedan comercializar a través de ellas sus productos y adquirir sus insumos (Altman, 2015), en mejores condiciones de realización (mejores precios, plazos, entre otros) y menos excluyentes que los tradiciones mecanismos de mercado (por ejemplo, intermediarios y grandes mayoristas).

Pero, además, en el caso exclusivo de la agricultura, para el autor antes citado las formas cooperativas constituyen (principalmente en las economías emergentes, aunque también en las más desarrolladas) un mecanismo para defender y preservar la independencia del pequeño agricultor o del agricultor familiar. Al mismo tiempo le proporcionan a este tipo de productores para permanecer en la actividad o hacerse más competitivas mediante el mejoramiento de la productividad y mayor calidad en sus productos $\mathrm{y} / \mathrm{o}$ servicios.

Esto añade un elemento crucial en la discusión, relacionado con la interrogante sobre qué tanto pueden las cooperativas sustituir a las organizaciones tradicionales (empresas verticales o burocráticas) como modelo de gestión, para mantenerse como unidades económicas realmente productivas $\mathrm{y}$ competitivas. Así, por ejemplo, plantea situaciones en las que, para mantener precios competitivos, probablemente deban sacrificar sus ingresos reales y con ellos, su nivel de vida y que conduzcan a estas familias a situaciones de pobreza. Adicionalmente hay todavía un elemento clave a considerar: normalmente las cooperativas no sobreviven si son mal gestionadas o no están construidas sobre un plan de negocios sostenible (Lukoschek et al., 2018).

Por lo tanto, los principios cooperativos han de constituir en las bases que permitan que un plan de negocios "robusto" sea exitoso (Altman, 2015).

Justamente aquí se halla el fundamento del hilo conductor del presente artículo: un modelo de organización basado en la horizontalidad puede ser más adecuado para enfrentar los desafíos del desarrollo rural, en la medida que considera sus distintas dimensiones y proporciona respuestas mejor adaptadas a las especificidades de cada localidad o territorio en el que se desenvuelve. Pero, al igual que sucede con la gestión de las organizaciones tradicionales, la misma debe estar fundamentada en un plan de negocios sólido y un adecuado manejo gerencial, que además orientado hacia la cohesión social/colectiva.

\section{Conclusiones}

La discusión a lo largo del artículo permite rescatar algunas ideas clave para cualquier estudio de las organizaciones en la actualidad. El primero de ellos es que en tanto agrupaciones humanas constituidas formalmente para alcanzar objetivos específicos, el manejo vertical de la autoridad es una realidad en un gran número de organizaciones; en particular, aquellas que están asociadas a lo que Marín (2013)

Álvarez Montalvo, Amparo 
llama "situs": empresas económicas, gobiernos, universidades $\mathrm{y}$ otras entidades dedicadas a investigación, complejos sociales y ejércitos. Dentro de las empresas económicas, además, históricamente el manejo vertical de la autoridad ha mostrado ser un modelo exitoso de gestión organizacional, por lo que se extendió rápidamente (adaptándolo a cada tamaño y necesidades de los mercados donde funcionan y de la propia organización en sí misma), desde las pequeñas empresas hasta las grandes corporaciones multinacionales.

No obstante sus demostradas ventajas, no dejan de tener algunas debilidades en su funcionamiento en la realidad. Por su carácter estático, rígido y mecanicista, basado en el cumplimiento de principios, reglas y normas (Melinkoff, 1986), el modelo vertical puede resultar muy eficiente en organizaciones cuyas actividades sean estandarizadas o repetitivas. De hecho, los postulados del modelo burocrático han coadyuvado con el posicionamiento de algunas de estas organizaciones en los mercados internacionales. Sin embargo, como modelo puede resultar poco flexible en realidades muy dinámicas y cambiantes, como ocurre con los espacios rurales.

En décadas recientes y particularmente tras la intensificación de las relaciones económicas globales, han aparecido otros modelos para la organización y gestión de las actividades de actores económicos, tanto en la economía en general como en el espacio rural, en particular. Las mismas han pretendido corregir o paliar las limitaciones impuestas por la rigidez de los modelos burocráticos y en mayor o menor medida han resultado ser una alternativa exitosa. Uno de estos modelos es el de las cooperativas, asociaciones autónomas y voluntarias de individuos, de propiedad conjunta, con una gestión democrática y orientadas a la consecución de objetivos colectivos sobre la base de los principios cooperativistas.

De éstos, son tres los que aportan las bases de la horizontalidad en tales organizaciones: la de adhesión voluntaria y abierta (que aunque existe en todas las organizaciones, en el asociacionismo solo ocurrirá en la práctica si el actor rural está convencido que la cooperativa realmente satisfará sus intereses y expectativas; en contraste, en las organizaciones tradicionales otras motivaciones como la pobreza rural o necesidades materiales insatisfechas podrían explicar su ingreso y permanencia en ellas); el control democrático de los miembros (las decisiones se toman en las asambleas de los asociados, siendo por tanto colectivas); y la autonomía e independencia (cada vez más las cooperativas, sin importar si son muy pequeñas o se trate de gigantes trasnacionales, contratan profesionales en áreas de gerencia, mercadeo, contabilidad y otras; todo ello con el fin último de garantizar el cumplimiento de sus objetivos pero también la sustentabilidad y permanencia en el tiempo). Gracias a ello el modelo organizativo en el contexto de la nueva ruralidad se torna más flexible, esto es, consigue una mayor capacidad de adaptación y para dar respuesta a las demandas del entorno dinámico y complejo en el que se desenvuelven. Esto a su vez da origen a modelos exitosos reseñados en la literatura, tales como los distritos agroindustriales, los territorios innovadores, entre otros.

En esta dinámica, la organización de agricultores/ productores primarios y la acción colectiva (que se materializa en las cooperativas) constituyen un mecanismo para mejorar el acceso de los agricultores a los mercados, para sustituir o mejorar la prestación de servicios públicos en los territorios rurales, para mejorar el acceso a los insumos, equipos y tecnologías requeridas en las actividades cotidianas, la coordinación horizontal (es decir, la integración en las llamadas cadenas de valor o cadenas agroproductivas), entre otras. Los desafíos en la nueva ruralidad pueden entonces convertirse en oportunidades a ser explotadas por este tipo de organizaciones, al tiempo que permitirán de consolidación de los procesos de desarrollo y cohesión social en los territorios en los que aquellas han de desenvolverse.

\section{Referencias bibliográficas}

Abramovay, R. (2006). Para una teoría de los estudios territoriales. En: Manzanal, M. y Neiman, G., Lattuada, M. (Comps.), Desarrollo rural. Organizaciones, instituciones y territorios (pp. 51-70). Buenos Aires: Ediciones Ciccus. 
Abramovay, R. (2007). Agricultura e desenvolvimento rural. Valor Econômico, (junho), A12-A12. Revisado el 15 de septiembre de 2018 en http://ricardoabramovay.com/ agricultura-e-desenvolvimento-rural/

Ahrne, G., Brunsso, N. y Seidl, D. (2017). On the fruitfulness of the concept of partial organization: A rejoinder to Apelt et al. European Management Journal, 35, 297-299. Revisado el 26 de diciembre de 2018 en https:// doi.org/10.1016/j.emj.2017.04.003

Alianza Cooperativa Internacional. (2013). Reglamento. Revisado el 08 de agosto de 2018 en http://ica.coop/sites/.../ICA\%20Bylaws\%20 -\%20updated\%202013\%20-\%20Spanish.pdf

Altman, M. (2015). Cooperative organizations as an engine of equitable rural economic development. Journal of Co-operative Organization and Management, 3, 14-23. https://doi.org/10.1016/j.jcom.2015.02.001

Baudel, M. (2001). A ruralidade no Brasil moderno. Por un pacto social pelo desenvolvimento rural (pp. 31- 44). En Giarracca, N. (Comp.), ¿Una nueva ruralidad en América Latina? Buenos Aires: Consejo Latinoamericano de Ciencias Sociales-CLACSO.

Beber, C. L., Theuvsen, L. y Otter, V. (2018). Organizational structures and the evolution of dairy cooperatives in Southern Brazil: A life cycle analysis. Journal of Co-Operative Organization and Management, 6(2), 64-77. doi:10.1016/j.jcom.2018.06.003

Chaddad, F. e Iliopoulos, C. (2012). Control rights, governance, and the costs of ownership in agricultural cooperatives. Agribusiness, 29(1), 3-22. doi:10.1002/agr.21328

Carneiro, M. J. (1998) Ruralidade: novas identidades em construção. Estudos Sociedade e Agricultura, 11, 53-75.

Carneiro, M. J. \& Teixeira, V. L. (2012). Para além das dualidades. En Carneiro, M. J. (Org.), Ruralidades Contemporâneas. Modos de viver e pensar o rural na sociedade brasileira (pp. 51-
66). Rio de Janeiro: Mauad.

Ceña, F. (1993). El desarrollo rural en sentido amplio. En Ramos, E. y Caldentey, P. (Eds.), El desarrollo rural andaluz a las puertas del siglo XXI (pp. 36-48). Sevilla: Junta de Andalucía.

Da Silva, G. (1996). A nova dinâmica da agricultura brasileira. (2a ed.). Campinas: UNICAMP.

Durkheim, E. (1968). Las reglas del método sociológico. Revisado el 28 de diciembre de 2018 en https://perio.unlp.edu.ar/catedras/ system/files/durkheim_emile_-_las_reglas_ del_metodo_sociologico_0.pdf

Etkin, J. (2005). Gestión de la complejidad en las organizaciones. Buenos Aires: Granica.

Ganga Contreras, F., Quiroz Castillo, J. y Maluk Uriguen, S. A. (2015). ¿Qué hay de nuevo en la teoría de agencia (TA)? Algunos trabajos teóricos y empíricos aplicados a las organizaciones. Prisma Social, 15, 685-707.

Gómez E., S. (2001). ¿Nueva Ruralidad? Un aporte al debate. Estudos Sociedade e Agricultura, (17), 5-32. Revisado el 26 de octubre de 2018 en http://bibliotecavirtual.clacso.org.ar/ar/ libros/brasil/cpda/estudos/dezessete/gomez17. htm

Hall, R. (1996). Organizaciones, estructuras, procesos y resultados. México, D.F.: Prentice Hall.

Hellin, J., Lundy, M. y Meijer, M. (2009). Farmer organization, collective action and market access in Meso-America. Food Policy, 34, 1622.

Johnston, B. F. y Mellor, J. W. (1962). El papel de la agricultura en el desarrollo económico. El Trimestre Económico, 29(114-2), 279-307. Revisado el 28 octubre de 2018 en https://www. jstor.org/stable/20855557

Juan F. Juliá-Igual, J. F., García-Martínez, G. y Meliá-Martí, E. (2011). Strategies developed by leading EU agri-food cooperatives in their growth model. Proceedings of the EAAE 2011

Álvarez Montalvo, Amparo

Estructuras Organizacionales y Nueva Ruralidad. El rol de las Cooperativas en el desarrollo territorial rural 
Congress Change and Uncertainty. Challenges for Agriculture, Food and Natural Resources (Zurich, August 30 to September 2). Revisado el 18 marzo de 2019 en https://ageconsearch. umn.edu/record/114453/files/Julia\%20Igual_ Juan_620.pdf

Ishikawa, K. (1986). ¿Qué es el control total de calidad? La modalidad japonesa. Bogotá: Norma.

Lukoschek, C. S., Gerlach, G., Stock, R. My Xin, K. (2018). Leading to sustainable organizational unit performance: Antecedents and outcomes of executives' dual innovation leadership. Journal of Business Research, 91, 266-276. doi:10.1016/j.jbusres.2018.07.003

Mariano, S. y Al-Arrayed, S. (2018). Combinations of absorptive capacity metaroutines: The role of organizational disruptions and time constraints. European Management Journal, 36(2), 171182. doi:10.1016/j.emj.2017.03.003

Marín, L. (2015). Sociología de las organizaciones. Influencia de las tecnologías de la información y la comunicación. Madrid: Editorial Fragua.

Melinkoff, R. (1986). La estructura de la organización. Los organigramas. Caracas: Contexto Editores.

Menanteau-Horta, D. y Marcuello-Servós, C. (2008). Una perspectiva sociológica para la acción social y el desarrollo: avances de la Sociocibernética. Revista Austral de Ciencias Sociales, 14, 111-124.

Morgan, G. (1990). Imágenes de la organización. Madrid: Editorial Ra-Ma.

Navarro, J., Roe, R. A. y Artiles, M. I. (2015). Taking time seriously: Changing practices and perspectives in Work/Organizational Psychology. Journal of Work and Organizational Psychology, 31, 135-145.

Ohno, Y. (1988). Toyota Production System. Beyond large-scale production. Portland: Productivity Press.
Pearce, R. J. (1997). Toward understanding joint venture performance and survival: A bargaining and influence approach to transaction to transaction cost theory. Academy of Management Review, 22(1), 203-225.

Pérez, E. (2001). Hacia una nueva visión de lo rural. En Giarracca, N. (Comp.), ¿Una nueva ruralidad en América Latina? (pp. 17-29). Buenos Aires: Consejo Latinoamericano de Ciencias SocialesCLACSO.

Perrow, C. (1991). Sociología de las organizaciones. Madrid: McGraw Hill.

Reig, E. (2007). Fundamentos económicos de la multifuncionalidad. En Gómez-Limón, J. A. y Barreiro Hurlé, J. (2007). La multifuncionalidad de la agricultura en España (pp. 19-39). Madrid: Eumedia S.A. y Ministerio de Agricultura, Pesca y Alimentación.

Retes L., R., Cruz B., F., Moreno M., S., Martín R., M. H., e Ibarra F., F. A. (2017). Desarrollo territorial: una propuesta de valor para el desarrollo de capacidades del capital humano en el distrito de desarrollo rural 142, Ures, Sonora. Revista Mexicana de Agronegocios, $38,241-252$.

Ritzer, G. (1996). La McDonalización de la sociedad. Un análisis de la racionalización en la vida cotidiana. Barcelona, España: Ariel.

Schejtman, A. y Berdegué, J. 2004. Desarrollo territorial rural. Santiago, Chile: Centro Latinoamericano para el Desarrollo RuralRimisp. Revisado el 03 de enero de 2019 en http://www.femica.org/noticias/docNoticias/ desarrolloterritorial.pdf

Silva Pérez, R. (2010). Multifuncionalidad agraria y territorio. Algunas reflexiones y propuestas de análisis. EURE, 36(109), 5-33.

Steiner, A. y Teasdale, S. (2017). Unlocking the potential of rural social enterprise. Journal of Rural Studies, XX. Revisado el 30 de octubre de 2018 en https://doi.org/10.1016/j. jrurstud.2017.12.021 
Tizon, P. (1996). Qu'est ce que le territoire? En Les territoires du quotidien, sous la direction de Guy Di Méo (pp. 17-34). Paris: L'Harmattan.

Tucker, D. A., Hendy, J. y Barlow, J. (2016). The dynamic nature of social accounts: An examination of how interpretive processes impact on account effectiveness. Journal of Business Research, 69(12), 6079-6087. doi:10.1016/j.jbusres.2016.06.014 